\title{
Mutual Coupling Reduction Using EBG in Steering Antennas
}

\author{
Gonzalo Expósito-Domínguez, José-Manuel Fernández-Gonzalez, Pablo Padilla, and \\ Manuel Sierra-Castañer
}

\begin{abstract}
In this letter, a dual circular polarized steering antenna for satellite communications in $\mathrm{X}$-band is presented. This antenna consists of printed elements grouped in an array, able to work from 7.25 up to $8.4 \mathrm{GHz}$ in both polarizations: left-handed circular polarization (LHCP) and right-handed circular polarization (RHCP). The module antenna is compact, with narrow beamwidth, and reaches a gain of $16 \mathrm{dBi}$. It has the capability to steer in elevation to $\pm 10^{\circ}$ and $\pm 40^{\circ}$ electronically with a Butler matrix. In order to reduce the mutual coupling between adjacent patches, electromagnetic band-gap (EBG) structures are introduced. These EBGs combine double-layer and edge location via in order to reduce the size, without changing the low-permittivity substrate, and therefore maintaining the high radiation efficiency of the antenna.
\end{abstract}

Index Terms-Electromagnetic band-gap (EBG) materials, mutual coupling reduction, Steering antennas.

\section{INTRODUCTION}

$\mathbf{N}$ OWADAYS, personal satellite communication systems are expected to provide wide variety of services using small g antennas [1], [2]. In order to get more flexible systems, the capability of mechanical or electrical steering is desired. The front ends in this type of systems are requested to be compact and lightweight [3]. In order to reduce the size of the antenna, high-permittivity substrates are used. However, with this type of substrates, not only the radiation efficiency of the antenna is reduced, but also the surface wave propagation modes are enhanced. This last effect leads into a strong mutual coupling between radiating elements in electrical steering systems when the scanning angle is not broadside and the phase feeding is $\alpha \neq 0^{\circ}$.

In this letter, a module of a wideband planar array antenna with dual circular polarization [left-handed circular polarization (LHCP) and right-handed circular polarization (RHCP)] and electrical elevation steering for satellite communication
TABLE I

ANTENNA SPECIFICATIONS

\begin{tabular}{|c|c|c|}
\hline Parameter & Value & Units \\
\hline \hline Frequency RX & $7.25-7.75$ & GHz. \\
\hline Frequency TX & $7.9-8.4$ & GHz. \\
\hline Polarization RX & LHCP* & $*$ Interchangeable. \\
Polarization TX & RHCP* & \\
\hline Gain & $\sim 16$ & dBi. \\
\hline Elevation steering & \pm 10 and \pm 40 & Degrees \\
\hline Dimensions & $<0.2$ & m. \\
\hline Antenna Efficiency & $>60$ & $\%$. \\
\hline Axial Ratio & $<3$ & $\mathrm{~dB}$. \\
\hline CP/XP & $>25$ & $\mathrm{~dB}$. \\
\hline Matching $S_{i i}$ & $>13$ & $\mathrm{~dB}$. \\
\hline Isolation $S_{i j}$ & $>15$ & $\mathrm{~dB}$. \\
\hline
\end{tabular}

systems is provided (Table I). In order to reduce the mutual coupling between radiating elements, electromagnetic band-gap (EBG) structures are introduced [4]. This letter is organized as follows. In Section II, double-layer edge location via EBG structures is introduced between two circular patches in order to study the effect of the mutual coupling reduction. In Section III, a prototype of a $4 \times 4$ array with steering capability to $\pm 10^{\circ}$ and $\pm 40^{\circ}$ by means of a Butler matrix network is designed, constructed, and fully measured. Finally, in Section IV the conclusions are drawn.

\section{Mutual Coupling Reduction}

In broadside antennas, it is relatively easy to obtain good matching for the radiating elements. However, this parameter changes very quickly when each row of elements is fed with a different phase shift $\alpha \neq 0^{\circ}$. This effect is due to the surface-wave propagation modes, which appear in thick substrates. In this particular case, in order to counteract this effect, cavities cannot be used due to the weight and cost constrains. Therefore, EBG structures are introduced between radiating elements [5]. Nevertheless, the separation of the radiating elements is $0.7 \lambda_{0}$, and the substrate permittivity is $\varepsilon_{r}=10.2$. These parameters lead into a big available space to place several rows of EBG structures, but reduce the radiating efficiency.

For the antenna under development, it is important to keep the radiation efficiency of the antenna, therefore a $\varepsilon_{\mathrm{r}}=2.17$ substrate is used. The available space between radiating elements under this circumstance is not enough to place original mushroom-shape EBGs. Therefore, multilayer and edge location via [6] techniques are combined to reduce the size of the mushrooms by $30 \%$. 

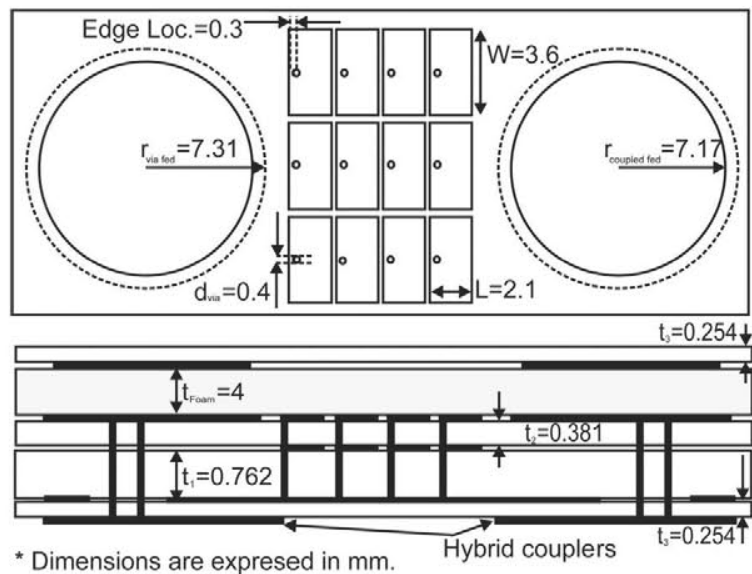

Fig. 1. Simulation scheme for mutual coupling reduction. The final dimensions are labeled.

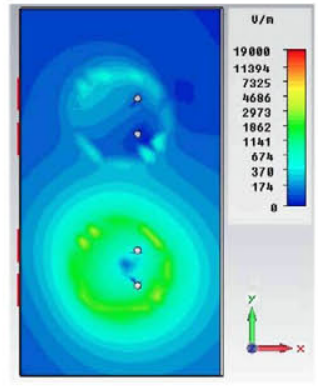

(a)

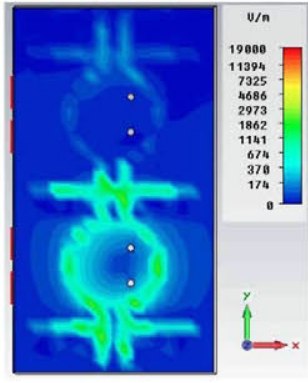

(b)
Fig. 2. $|\mathrm{E}|$-field simulation of two circular patches with dual circular polarization at $7.825 \mathrm{GHz}$. The fields represented in the figures correspond to the bottom patch (via fed patches). (a) Without EBG structures. (b) With EBG structures.

In order to prove the effectiveness of these electromagnetic barriers, four rows of double-layered edge-located via EBG mushrooms (F-shape) are introduced between two circular patches with double circular polarization. The elements are fed by a $90^{\circ} / 3-\mathrm{dB}$ branch-line couplerp in order to get the double circular polarization (Fig. 1).

These two circular patches with and without EBG F-shape mushroom are built. These printed antennas, separated $0.6 \lambda_{0}$, are mounted with double stacked patch with permittivity of $\varepsilon_{\mathrm{r}}=$ 2.17 and a foam layer between them of $4 \mathrm{~mm}$ in order to cover the whole bandwidth $(20 \%)$. The thicknesses of the substrates are $1.143 \mathrm{~mm}$ for the bottom patch (via fed patch) and $0.254 \mathrm{~mm}$ for the upper patch and the feeding network. The dimensions for the EBG structures are $3.6 \mathrm{~mm}$ for the horizontal axis and $2.1 \mathrm{~mm}$ for the vertical axis. The design method in order to calculate these dimensions is based on the $L C$ equivalent circuit and optimized with an electromagnetic (EM) simulator [7].

In Fig. 2(a), |E -field simulations for the two patch antennas with and without EBG structures are shown for LHCP. In Fig. 2(b), it can be seen graphically how $|\mathrm{E}|$-field value decays deeper when using EBG structures in the middle of the frequency band-gap.

These antennas are constructed, and a comparison between simulations and measurements of $S$-parameters for a $2 \times 1$ array antenna is presented in Fig. 3. LHCP ports are excited, and

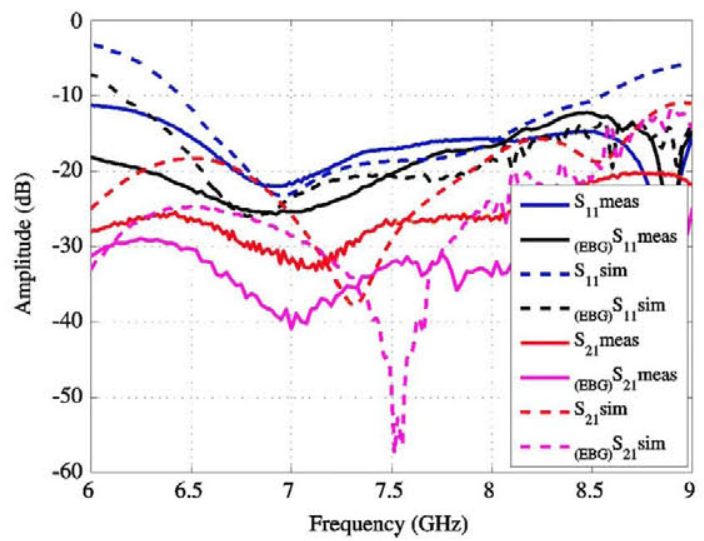

Fig. 3. Comparison of measurements and simulated $S$-parameters for $2 \times 1$ array. Measurements are presented in continuous line, and simulations in dashed line.

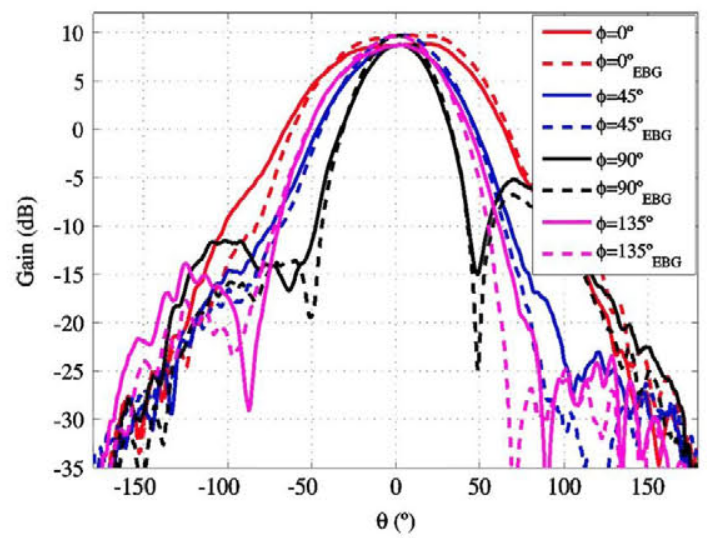

Fig. 4. Radiation patterns of $2 \times 1$ test patch antenna array without (continuous lines) and with (dashed lines) EBG structures are presented.

RHCP ports are loaded with $50-\Omega$ loads. Similar behavior between simulations and measurements is achieved. There is a mutual coupling reduction between the two patch antennas of approximately $5 \mathrm{~dB}$ and an improvement of reflection coefficient $S_{i i}$ of $\sim 3 \mathrm{~dB}$ in most of the operation band. This coupling reduction remains for the different phase feedings of the elements.

In Fig. 4, the radiation patterns for $\phi$ cuts are presented. It can be noticed that for the cuts $\phi=45^{\circ}, 90^{\circ}$, and $135^{\circ}$ that the beamwidth is reduced for the EBG case. EBG structures surrounding microstrip antennas tend to make narrower the radiation pattern of the radiating element; this beamwidth reduction increases the directivity of the main lobe of the antenna since the effect of the EGB barriers is similar to the effect of cavities. On the other hand, due to the structures placed on the edge of the antenna, $\sim 5 \mathrm{~dB}$ of back lobe reduction is obtained. Radiation pattern for $\phi=90^{\circ}$ presents asymmetry due to the measurement setup in the anechoic chamber.

\section{Design And Measurements}

The maximum separation between elements for avoiding grating lobes in steering antennas is a double problem. First, by placing the radiating elements very close, $|E|$-field interaction between radiating elements is stronger. In the second place, the 


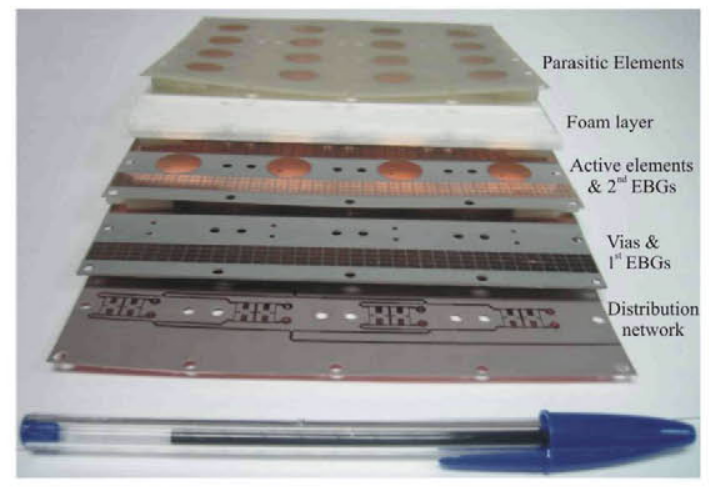

Fig. 5. Layer view of the $4 \times 4$ array with $\mathrm{EBG}$ structures construction.

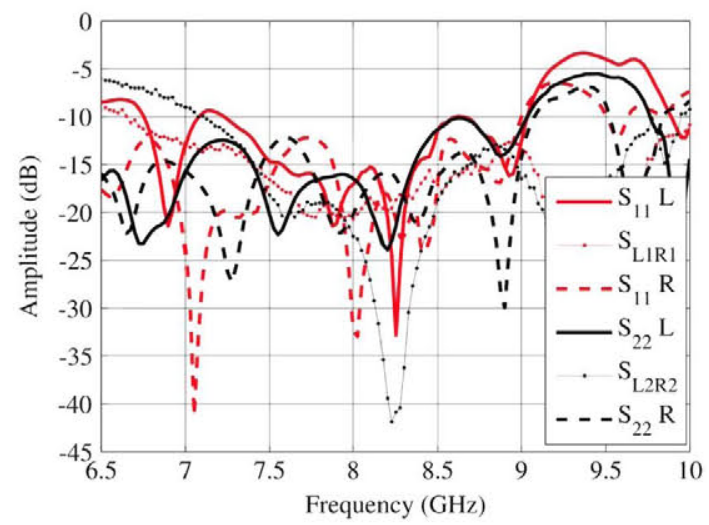

Fig. 6. $4 \times 4$ array with EBG structures $S$-parameters measurements.

effective area of the antenna is reduced. For this satellite application, broadband capability is required, and thick substrate is used. Thus, the surface-wave propagation modes are enhanced, and the mutual coupling between elements grows. For that reason, the steerable antenna prototype from [8] has been built to measure the radiation patterns and to verify the features and performances when mushroom F-shape structures are introduced between elements in order to suppress surface-wave propagation and, consequently, to reduce mutual coupling.

In Fig. 5, the printed array layers are presented. In the bottom layer $(0.254 \mathrm{~mm}$ thick), the feeding distribution network and $90^{\circ} / 3-\mathrm{dB}$ hybrid couplers are printed. In the second layer (0.762 $\mathrm{mm}$ thick), the first mushrooms and the vias for the patches are placed. On top of it, the third layer $(0.381 \mathrm{~mm}$ thick) contains the via-fed circular patches and second the mushrooms, which are stacked with the first mushrooms in the previous layer. On the top layer $(0.254 \mathrm{~mm}$ thick), parasitic fed patches are printed. Finally, between via feed patches and parasitic fed patches, a 4-mm foam layer is placed in order to enhance the bandwidth [9]. The distance between the radiating elements is $0.6 \lambda_{0}$ for vertical axis in order to avoid grating lobes when electronic steering is used. However, for horizontal axis, $0.85 \lambda_{0}$ is used in order to reduce the number of elements and to get the required directivity.

Fig. 6 presents the measurements for eight input ports of the 16-elements array. The continuous lines show the left-side ports, meanwhile the dashed lines represent the right-side ports. The marked lines correspond to the isolation between two closer

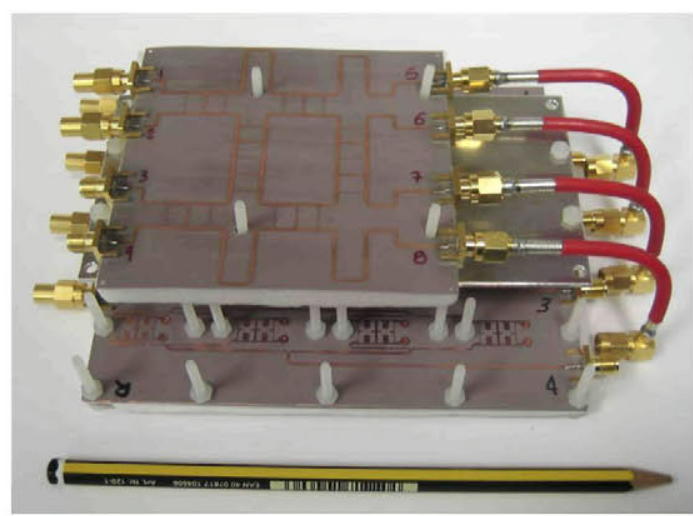

Fig. 7. $4 \times 4$ array and Butler matrix network connection for LHCP configuration.

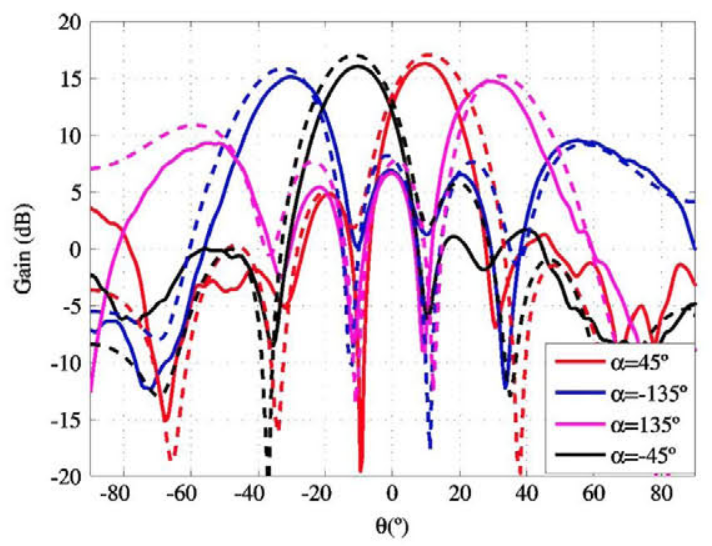

Fig. $8.4 \times 4$ steering array radiation pattern for $\mathrm{RHCP}$, at the center frequency $(7.825 \mathrm{GHz})$.

ports. The measured isolation is adequate enough with a value below $15 \mathrm{~dB}$ for the whole band, and the measured reflection coefficient for all the ports is below $13 \mathrm{~dB}$, thanks to the EBG structures; otherwise, these values do not reach $10 \mathrm{~dB}$.

In order to get the beam steering, a passive Butler matrix network is constructed [10]. If the Butler network, in Fig. 7, is connected to left-side ports, the antenna radiation will be LHCP, and vice versa. The rest of the ports are loaded with $50-\Omega$ loads. Butler matrix network yields $-45^{\circ},+135^{\circ},-135^{\circ}$, and $+45^{\circ}$ phase shift between output ports when exciting ports $1-4$, respectively.

These phase shifts between elements $\left(\alpha= \pm 45^{\circ}\right.$ and $\left.\pm 135^{\circ}\right)$ are used to calculate the pointing directions of the antenna. Those directions are calculated by applying

$$
\left.\theta\right|_{\psi=0}=\arccos \left(\frac{-\alpha}{k d}\right)
$$

where $\psi=0$ is the maximum directivity, $k$ is the wavenumber, and $d$ is the physical distance between elements (in this case $0.6 \lambda_{0}$ ). The steering directions for the antenna, $+10^{\circ},-40^{\circ}$, $+40^{\circ}$, and $-10^{\circ}$, are obtained.

In Fig. 8, the steering vertical plane radiation patterns for RHCP at the center frequency $(7.825 \mathrm{GHz})$ and different pointing directions are presented. Good agreement between simulations (dashed lines) and measurements (continuous lines) is obtained. CP/XP ratio is better than $15 \mathrm{~dB}$. Due to the 


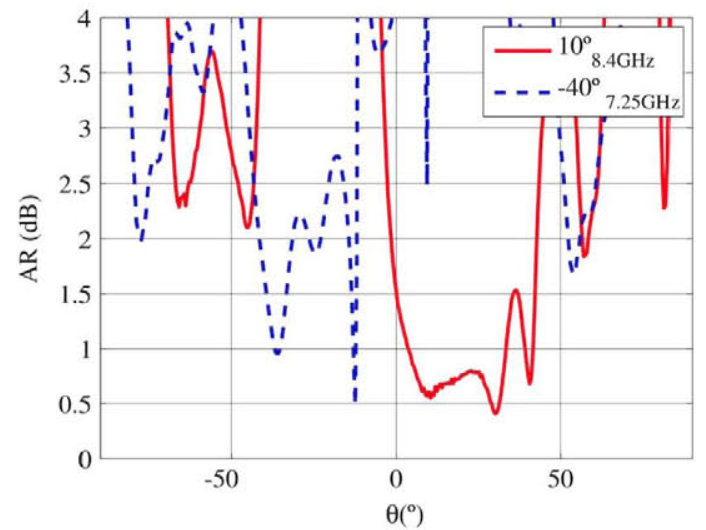

(a)

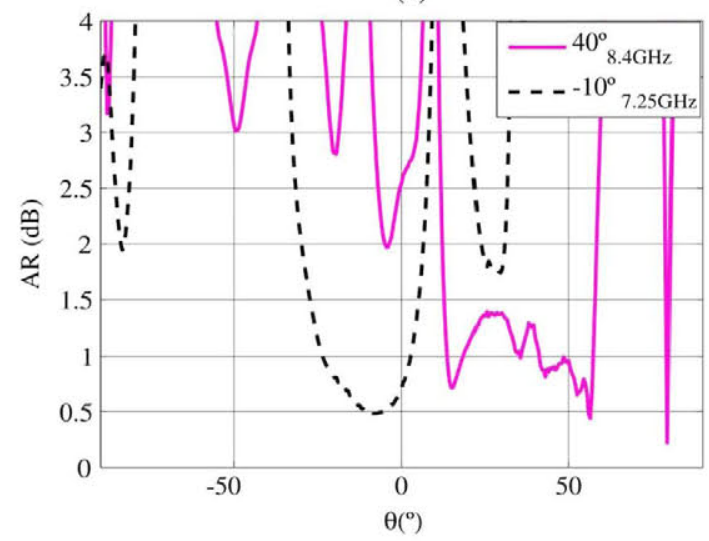

(b)

Fig. 9. $4 \times 4$ steering array axial ratio for frequencies 7.25 and $8.4 \mathrm{GHz}, \mathrm{RHCP}$ and LHCP over the scanning angles. (a) $+10^{\circ}$ LHCP and $-40^{\circ}$ RHCP beams. (b) $-10^{\circ} \mathrm{LHCP}$ and $+40^{\circ} \mathrm{RHCP}$ beams.

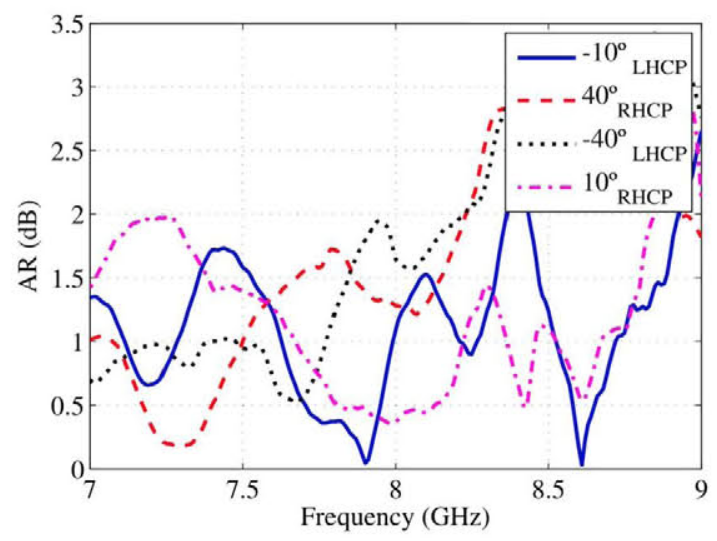

Fig. 10. $4 \times 4$ steering array axial ratio for RHCP and LHCP and different pointing directions over the working frequency.

uniform distribution, sidelobe level (SLL) is under $12 \mathrm{~dB}$ for $\pm 10^{\circ}$ and under $7 \mathrm{~dB}$ for $\pm 40^{\circ}$ beams.

Finally, in Fig. 9(a) and (b), the axial ratio for the different beams and different steering angles at the end and the beginning of the frequency band are shown. In this case, different frequencies, polarizations, and steering directions are shown in order to prove the good features of the antenna.

As it was expected, the introduction of EBG structures between the radiating elements has no significant influence in the circular polarization of the antenna, and only slight differences are appreciated. Finally, in Fig. 10, the axial ratio of the antenna is shown, related to the frequency. The purity of the polarization is better than $3 \mathrm{~dB}$ over the whole frequency band.

\section{CONCLUSION}

This letter presents and proposes a wideband planar array antenna in X-band with dual circular polarization and electrical elevation steering for satellite communication systems. The array antenna is built in low-permittivity substrate to enhance the radiation efficiency of the antenna. In order to reduce the mutual coupling, EBG structures are introduced between the radiating elements. The available space between the radiating elements $\left(0.6 \lambda_{0}\right)$ is very small, and therefore double-layer and via-edge location techniques are combined to reduce the size of the mushrooms. With these techniques, an increase of $\sim 5 \mathrm{~dB}$ isolation is obtained, and we observe a reduction of the beamwidth in the main lobe, which enhances the antenna directivity. Finally, the electromagnetic barriers placed at the edges of the $4 \times 4$ array reduce the antenna back lobe without interfering with the behavior of the electrical steering antenna.

The main advantage of this technique is the use of planar technology and the possibility of full integration in planar antennas without the necessity of cavities for the radiating elements.

\section{ACKNOWLEDGMENT}

The simulations contained in this work have been carried out using CST Microwave Studio Suite 2011 under a cooperation agreement between Computer Simulation Technology (CST) and the Universidad Politécnica de Madrid. The authors kindly thank the company NELTEC S.A. for giving samples of the substrates, in which the prototypes were built. They also thank to the ETSI de Telecomunicación for the economical support.

\section{REFERENCES}

[1] J. Evans, "Satellite systems for personal communications," IEEE Antennas Propag. Mag., vol. 39, no. 3, pp. 7-20, Jun. 1997.

[2] M.-A. Salas-Natera, A. García-Aguilar, J. Mora-Cuevas, J.-M. Fernández-González, P. Padilla, J. García-Gasco, R. Martínez-Rodríguez, M. Sierra-Pérez, L. de Haro-Ariet, and M. Sierra-Castañer, Satellite Communications. Rijeka, Croatia: InTech, Jul. 2011.

[3] J. M. Fernández-González, P. Padilla, G. Expósito-Domínguez, and M. Sierra-Castañer, "Lightweight portable planar slot array antenna for satellite communications in X-band,"IEEE Antennas Wireless Propag. Lett., vol. 10, pp. 1409-1412, 2011.

[4] D. Sievenpiper, L. Zhang, R. F. J. Broas, N. G. Alexópolous, and E. Yablonovitch, "High-impedance electromagnetic surfaces with a forbidden frequency band," IEEE Trans. Microw. Theory Tech., vol. 47, no. 11, pp. 2059-2074, Nov. 1999.

[5] F. Yang and Y. Rahmat-Samii, "Microstrip antennas integrated with electromagnetic band-gap (EBG) structures: A low mutual coupling design for array applications," IEEE Trans. Antennas Propag., vol. 51, no. 10, pp. 2936-2946, Oct. 2003.

[6] E. Rajo-Iglesias, L. Inclán-Sanchez, J.-L. Vázquez-Roy, and E. Garcia-Muñoz, "Size reduction of mushroom-type EBG surfaces by using edge-located vias," IEEE Microw. Wireless Compon. Lett., vol. 17, no. 9, pp. $670-672$, Sep. 2007.

[7] G. Expósito-Domínguez, J. M. Fernández-González, P. Padilla, and M. Sierra-Castañer, "EBG size reduction for low permittivity substrates," Int. J. Antennas Propag., 2012, submitted for publication.

[8] G. Expósito-Domínguez, J. M. Fernández-González, P. Padilla, and M. S. Castañer, "Dual circular polarized steering antenna for satellite communications in x band," Prog. Electromagn. Res., vol. 122, pp. 61-76, 2012.

[9] R. Garg, I. bahl, and A. Ittipiboon, Microstrip Antennas Design Handbook. Norwood, MA: Artech House, 2001.

[10] J. Butler and R. Lowe, "Beam-forming matrix simplifies design of electronically scanned antennas," Electron. Des., vol. 9, pp. 170-173, 1961. 